POS PROCEEDINGS

\title{
The Italian Polarimetry Programmes
}

\author{
Enrico Costa ${ }^{a}$, Ronaldo Bellazzini ${ }^{b}$, Giorgio Matt ${ }^{c}$, Gianpiero Tagliaferri ${ }^{d}$, Stefano \\ Basso $^{d}$, Alessandro $\mathrm{Brez}^{b}$, Vincenzo Cotroneo ${ }^{d}$, Sergio Di Cosimo ${ }^{a}$, Francesco \\ Lazzarotto $^{a}$, Massimo Minuti ${ }^{b}$, Fabio Muleri $^{a}$, Giovanni Pareschi ${ }^{d}$, Michele \\ Pinchera $^{b}$, Alda Rubini ${ }^{a}$, Paolo Soffitta ${ }^{a}$, Gloria Spandre ${ }^{b}$ \\ ${ }^{a}$ INAF/IASF Rome \\ Via del Fosso del Cavaliere 100, I-00133 Rome, Italy \\ ${ }^{c}$ INFN Pisa \\ Largo B. Pontecorvo 3, I-56127 Pisa, Italy \\ "Universitá degli studi "Roma Tre" \\ Via della Vasca Navale 84, I-00146 Rome \\ Largo San Leonardo Murialdo, 1, I-00146 Roma \\ ${ }^{d} I N A F / O A B$ \\ Via E. Bianchi 46, I-20121 Milano
}

E-mail: enrico.costa@iasf-roma.inaf.it

The Gas Pixel Detector is a new generation X-ray polarimeter based on photoelectric effect that has been developed by INFN of Pisa and INAF/IASF of Rome. Its expected performances have been recently supported by the measurement of the modulation factor at a few $\mathrm{keV}$, namely in the energy range of maximum interest. About $80 \%$ of the sensitivity expected on the basis of a Monte Carlo software are achieved with the current prototype, hence definitely proving the feasibility of $\mathrm{X}$-ray photoelectric polarimetry with the GPD. Its advanced status of development allows to plan its use in the near future space missions and three proposals have passed a certain level of selection by the Italian and European space agencies. Two scenarios have emerged: the first is a small pathfinder mission, able to measure the polarization of galactic and bright extragalactic sources in a few days of observation with a low-cost X-ray telescope, while the second is the introduction of this device in the focal plane of a large international observatory, which would make accessible even the faint extragalactic sources. Here we briefly summarize both these opportunities.

Polarimetry days in Rome: Crab status, theory and prospects

October 16-17, 2008

Rome, Italy 


\section{Introduction}

Polarimetry is the branch of X-ray astronomy where there is the largest gap between expectations and actual results. It has been recognized as a primary tool in the investigation of compact sources since the beginning of X-ray astronomy $[1 ; 2]$, however only meagre results have been achieved due to the lack of sensitivity of the X-ray polarimeters which flew. As a matter of fact, it has been possible to register the positive detection of X-ray polarization only in the emission of the Crab nebula [3; 4]. The lack of experimental feedback has not prevented the development of a rich literature from which we expect that almost all galactic and extragalactic sources should emit partially polarized radiation (for a recent review, see [5]).

The Gas Pixel Detector (GPD hereafter) is a completely Italian project developed by INFN of Pisa and INAF/IASF of Rome and consists in a new generation polarimeter based on photoelectric absorption $[6 ; 7 ; 8]$. The GPD is basically composed by a cell gas where photons are absorbed, a Gas Electron Multiplier and a fine-subdivided ASIC detector: its 105000 pixels work as many proportional counters but their small dimension, $50 \mu \mathrm{m}$, allows to accurately resolve the tracks of photoelectrons. Their initial directions of emission carry a significant memory of the polarization of the absorbed photons.

No major issues are expected in the use of the instrument in space. This device is fundamentally composed by $105 \times 10^{3}$ independent proportional counters with a common gas volume. The possibility of destructive discharges is limited thanks to the low background of the detector, which allows a low gain of the GEM, and the most critical issue remains the pollution of the gas mixture. However a sealed version of the GPD has been working in laboratory for more than 15 months without showing any degradation of the performances. The GPD has also survived without any degradation after the irradiation of iron ions corresponding to several years in orbit performed in the Heavy Ion Medical Accelerator in Chiba (HIMAC) and to vibrations and thermo-vacuum tests between -15 and $45^{\circ} \mathrm{C}$. The current prototype used in the laboratory weights $1.6 \mathrm{~kg}$ and it is contained in a box $140 \times 190 \times 70 \mathrm{~mm}^{3}$, including read-out electronics.

The advanced status of development of the GPD makes possible its use with a grazing incidence optics on-board future missions in a few years. Its high sensitivity allows to design small and low cost missions which would anyway unveil the X-ray polarimetry for galactic and bright extragalactic sources. Subsequent larger missions could then be optimize for the scientific questions arisen by these pathfinder missions. Below we summarize the perspective of measurement of the GPD in the context of Italian missions (see Table 1).

\section{Pathfinder missions: PolariX and EXP'}

Pathfinder missions are intended as low cost and small missions to launch in a few years: they are designed to be very versatile and allow an exploration of the rich variety of scientific objectives of X-ray polarimetry. The baseline is the use of a He-DME mixture, which allows a response in the 2-10 keV energy range, with good timing $(8 \mu \mathrm{s})$ and spectral capabilities (20\% at $6 \mathrm{keV})$. The short focal length (2.1-3.5 m) limits the angular resolution of the instrument (because of the inclined penetration of photons in the gas cell of the GPD) to a few tens of arcsec (see Table 1). 


\begin{tabular}{lcccccccc}
\multicolumn{1}{c}{$\#$} & $\begin{array}{c}f \\
\mathrm{~cm}\end{array}$ & $\begin{array}{c}\text { Ang. Res. } \\
\operatorname{arcsec}\end{array}$ & $\begin{array}{c}\text { fov } \\
\operatorname{arcmin}^{2}\end{array}$ & $\begin{array}{c}E \\
\mathrm{keV}\end{array}$ & $\begin{array}{c}\Delta E \\
\mathrm{keV} \text { at 6 keV }\end{array}$ & $\begin{array}{c}\tau \\
\mu s\end{array}$ & $\begin{array}{c}T \\
\mathrm{ks}\end{array}$ \\
\hline \hline Pathfinder missions & & & & & & & & \\
\hline PolariX & 3 & 350 & 24 & $\sim 15 \times 15$ & $2-10$ & $20 \%$ & 8 & 15.7 \\
EXP $^{2}$ /HXMT & 2 & 210 & 40 & $\sim 25 \times 25$ & $2-10$ & $20 \%$ & 8 & 10.7 \\
\hline \hline Large observatory & & & & & & \\
\hline XPOL/IXO 1 & $2000-2500$ & 5 & $\sim 2.6 \times 2.6$ & $2-10$ & $20 \%$ & 8 & 0.75
\end{tabular}

Table 1: Comparison of the main characteristics of the X-ray photoelectric polarimeters based on the GPD and inserted in next satellite missions. \# is the number of telescopes, $f$ the focal length, Ang. Res. the angular resolution (HEW), $E$ the energy range, $\Delta E$ the spectral resolution, $\tau$ the time resolution. Eventually $T$ is the time required for reaching an MDP of $1 \%$ for the Crab nebula between 2 and $10 \mathrm{keV}$.

PolariX (Polarimetro $X$, which in Italian means "X-ray polarimeter", [9]) is a small mission dedicated to X-ray polarimetry proposed to the Italian space agency (ASI, Agenzia Spaziale Italiana) and accepted for a Phase A study which ended in December 2008. PolariX is currently waiting for the downselection to two approved missions and, if selected, it will be launched in mid 2014. It is developed under the responsibility of IASF/INAF of Rome with the contribution of INFN of Pisa (for the development of the focal plane) and the scientific support of the University of Rome "Roma Tre". The ambitious schedule of PolariX and the small budget allocate to small missions (50 millions of euro) requires the use of technologies with high degree of readiness. With this concept in mind, we plan the use of three telescopes of the mission Jet- $X$ (which has never flown), which would be integrated by OAB/INAF, and to exploit the heritage of the spacecraft of SIMBOL-X, planned to be built by Thales Alenia Space of Turin. The ground segment is instead managed by the Telespazio of Rome on the basis of that of the Italian mission AGILE [10].

An exploded view of PolariX is reported in Fig. 1(a). PolariX is designed to host three telescopes: however, since the mandrels of the Jet-X optics are still available, two further units could be built and easily inserted in the payload with a modest cost. Note that the use of a cluster of small and low cost telescopes allows to reach in practice the same sensitivity as a single telescope with equivalent total area, since virtually any source accessible to X-ray polarimetry will be much stronger than internal and diffuse background.

The focal plane (see Fig. 1(b)) hosts in the baseline three detectors, namely as much as the optics. A baffle protects the GPD by straight light and an electrostatic grid on the top prevent that charged particles overload the HV power supply. The calibration of each detector is performed in-flight thanks to unpolarized sources $\left(\mathrm{Fe}^{55}\right.$ and $\mathrm{Cd}^{109}$ ) and a polarized one (at 2.7 and $5.9 \mathrm{keV}$ ) based on Bragg diffraction at nearly 45 degrees [11]. They are mounted on filter wheel which also provides a close position (for the launch and for the measurement of the internal background), a gray filter (for very bright sources) and a diaphragms (to exclude bright objects in the field of view of faint sources). At any time, the sky orthogonal to the direction of the $\operatorname{Sun} \pm 20^{\circ}$ is accessible to PolariX.

$E X P^{2}$ (Efficient X-ray Photoelectric Polarimeter, [12]) is the Italian contribution approved by ASI to the Chinese mission HXMT (Hard X-ray Modulation Telescope), devoted to a sensitive survey in hard X-rays and to pointed observations for an half of observation time. Two similar 


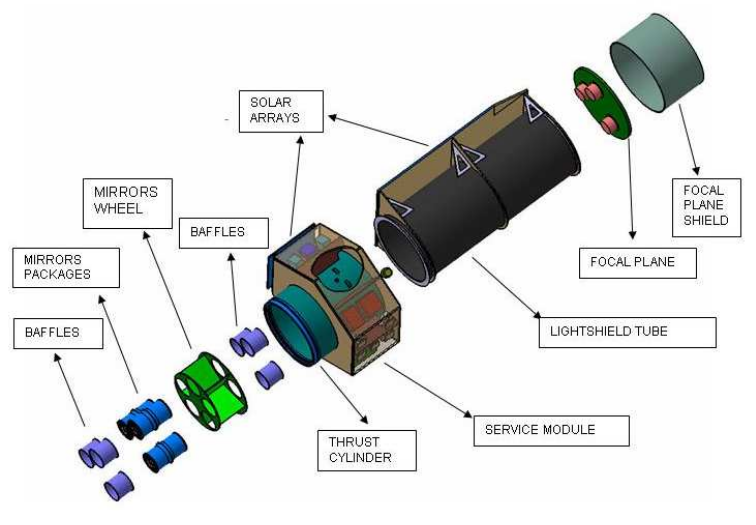

(a)

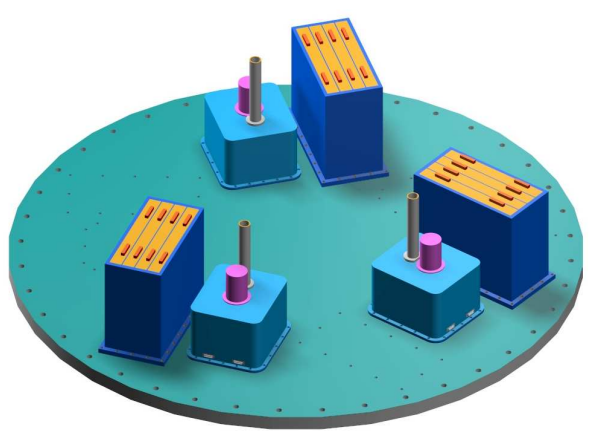

(b)

Figure 1: (a) Exploded view of PolariX (image credit: Thales Alenia Space of Turin). (b) Layout of the focal plane. The box which contains the HV power supplies and the read-out electronics is in orange (images credit: INFN of Pisa).

instruments, possibly tuned to slightly different energy bands, are placed in the focal plane of as much as X-ray telescopes built by OAB/INAF, the latter being mounted on the side of the HXMT spacecraft. The focal plane layout is similar to that of PolariX, with a filter wheel which allows the periodic monitoring of the performances of the detector.

\subsection{XPOL on-board IXO/XEUS}

A polarimeter based on the GPD (XPOL, $X$-ray Polarimeter) is also inserted in the focal plane of the International X-ray Observatory (IXO) space mission, to be launched in 2020. Even in this case, the instrument should be filled with a Helium-DME mixture sensitive between 2 and $10 \mathrm{keV}$, even if further studies could allow to extend these limits to fully exploit the large area of the telescope, specially below $2 \mathrm{keV}$. The current version of the GPD [7] can't sustain the rate expected for strong sources (a few thousands of count per second in the case of the Crab Nebula) and a re-design of the ASIC chip is required to increase the read-out clock.

XPOL gains more than an order of magnitude of sensitivity with respect to pathfinder missions, allowing a survey of even faint extragalactic sources in a reasonable observation time. The long focal length of the IXO telescope $(20-25 \mathrm{~m})$ is another scientific driver of this mission: the angular resolution of polarimeter would be 5 arcsec (including the blurring caused by the inclined penetration in the gas cell), opening the way to finely resolve diffuse sources like Pulsar Wind Nebulae or galactic and extragalactic jets.

\section{Scientific performances}

The scientific performances of a pathfinder mission (PolariX is taken as example) and XPOL are summarized in Fig. 2(a) and Fig. 2(b) respectively, where the observation time to reach a certain 


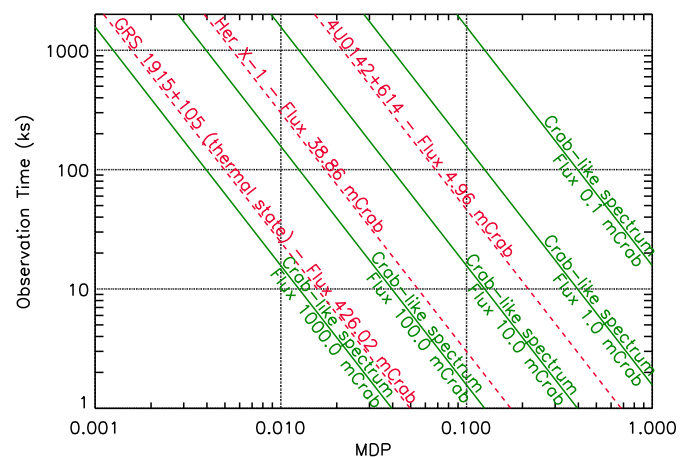

(a)

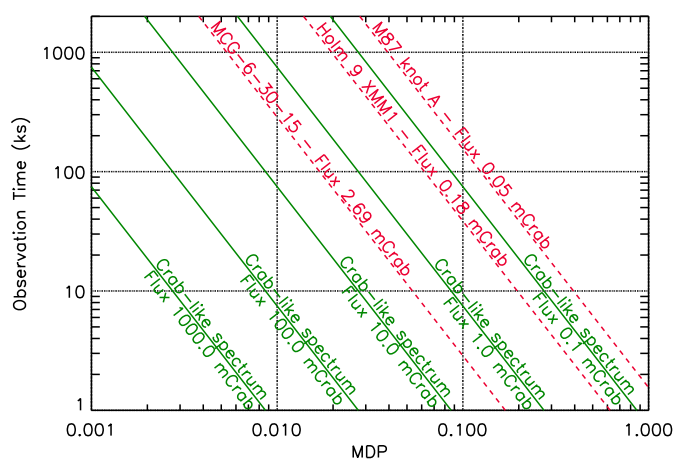

(b)

Figure 2: Observation time required to reach a certain MDP as a function of the flux of the source for PolariX (a) and XPOL (b).

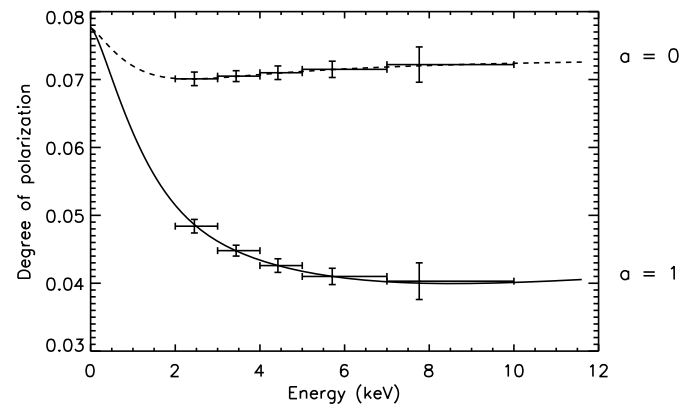

(a)

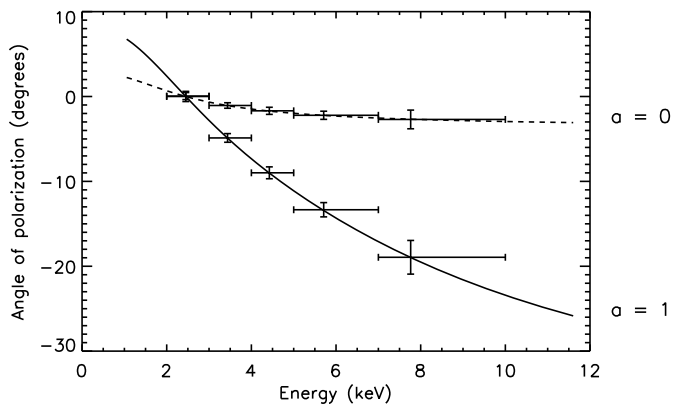

(b)

Figure 3: Simulated 1 Ms observation of GRS 1915+105 with PolariX. The energy dependence of the degree and of angle of polarization are in (a) and (b). The solid and dashed curves refer to the case of a Kerr and a Schwarzschild black hole respectively. $\tau=1.0$ and $\theta_{0}=70^{\circ}$. Errors are at the 1- $\sigma$ level [14].

Minimum Detectable Polarization (MDP) at the 99\% confidence level is reported as a function of the flux. Reasonable observation time with the small missions will be up to $1 \mathrm{Ms}$, i.e. about 10 days, while in the IXO scenarios pointings up to a few days are expected.

The possibilities of X-ray polarimeters on-board next missions can be better appreciated with a specific scientific case. It's a long-standing prediction that the measurement the direction of polarization of thermal emission from the accretion disk around galactic black holes is a powerful probe to investigate the spin of the compact object [13]. Recent fully relativistic simulations [14] shows that this effect is observable even with PolariX and a long pointing (see Fig. 3)

\section{Conclusion}

In a few years an X-ray photoelectric polarimeter based on the GPD could be launched as a 
dedicated mission (PolariX) and on-board the Chinese satellite HXMT (EXP2). These pathfinders would open the way for the first time to the polarimetry of several tens of galactic and bright extragalactic sources. Refined studies of the most interesting objects emerged by this survey and the polarimetry of faint extragalactic sources would be accessible to XPOL, the polarimeter onboard IXO to be launched in 2020 .

\section{References}

[1] R. Novick. Stellar and Solar X-Ray Polarimetry. Space Science Reviews, 18:389, 1975.

[2] M. J. Rees. Expected polarization properties of binary X-ray sources. MNRAS, 171:457, 1975.

[3] M. C. Weisskopf et al. A precision measurement of the X-ray polarization of the Crab Nebula without pulsar contamination. ApJ, 220:L117, 1978.

[4] A. J. Dean et al. Polarized Gamma-Ray Emission from the Crab. Science, 321:1183, 2008.

[5] M. C. Weisskopf et al. The prospects for X-ray polarimetry and its potential use for understanding neutron stars. Paper Presented at the 363rd Heraeus Seminar in Bad Honef, Germany. Springer Lecture Notes, submitted, 2006.

[6] E. Costa et al. An efficient photoelectric X-ray polarimeter for the study of black holes and neutron stars. Nature, 411:662, 2001.

[7] R. Bellazzini et al. Direct reading of charge multipliers with a self-triggering CMOS analog chip with $105 \mathrm{k}$ pixels at $50 \mu \mathrm{m}$ pitch. Nuclear Instruments and Methods in Physics Research A, 566:552, 2006.

[8] R. Bellazzini et al. A sealed Gas Pixel Detector for X-ray astronomy. Nuclear Instruments and Methods in Physics Research A, 579:853, 2007.

[9] E. Costa et al. POLARIX: a small mission of x-ray polarimetry. In Proc. of SPIE, volume 6266, page 62660R, 2006.

[10] M. Tavani et al. The AGILE space mission. Nuclear Instruments and Methods in Physics Research A, 588:52, 2008.

[11] F. Muleri et al. A very compact polarizer for an X-ray polarimeter calibration. In Proc. of SPIE, volume 6686, page 668610, 2007.

[12] P. Soffitta et al. X-ray polarimetry on-board HXMT. In Proc. of SPIE, volume 7011, pages 701128-1, 2008.

[13] P. A. Connors et al. Polarization features of X-ray radiation emitted near black holes. ApJ, 235:224, 1980.

[14] M. Dovčiak et al. Thermal disc emission from a rotating black hole: X-ray polarization signatures. MNRAS, 391:32, 2008. 